Perspectives and Debates

\title{
COVID-19 pandemic: Emerging perspectives and future trends
}

\author{
Syed Amin Tabish
}

Sher-i-Kashmir Institute of Medical Sciences, Srinagar, India

\begin{abstract}
World is living on the edge. The human cost of COVID pandemic could be extraordinary. We find ourselves in a time of great economic, social, and medical uncertainty. The pandemic demands action on many fronts, from prevention to testing to treatment. We need to create simple, cheap, more accessible testing for SARS-CoV-2. A faster way has to be developed to identify antibodies that neutralize the virus. More than 100 vaccines for the SARS-CoV-2 are at various stages of development. Some six groups have already begun injecting formulations into volunteers in safety trials; others have started testing in animals. The biggest challenge is to determine which vaccine is ideal. Reason and science have to guide us. There is urgent need to critically appraise evidence in deciding how to treat patients. We need a drug or combination of drugs that work. Remdesivir has generated hope. It may prove to be a magic bullet. Countries like Taiwan, Vietnam, Singapore, Hong Kong, South Korea, New Zealand have done exceptionally well to contain the spread of COVID-19. It is widely believed that during the pandemic treatment suffers. Patients with diseases like cancer, diabetes, renal failure, CAD and pregnant women need special attention. As the pandemic pushes up levels of hunger among the global poor, governments must prevent devastating nutrition and health consequences for children missing out on school meals amid school closures. Nations will have endemic SARS-CoV-2 infection for the foreseeable future. A structured and well-coordinated approach is critical for tackling this global crisis.
\end{abstract}

\section{Introduction}

Human population faces a grave health-related challenge in the form of infectious diseases that are responsible for the highest number of deaths globally. The tiniest primitive invisible life form is now controlling the behavior of the most powerful and evolved life form on earth. Tough times ahead! 1,2

Between 1940 and 2020, around 340 new infectious diseases have emerged globally. The compromised health and disability due to infectious diseases, which accounts for $30 \%$ of all disabili- ty-adjusted life years, decreases work productivity, and increases morbidity. ${ }^{1,2}$ Since the dawn of civilization, humans and microbes have co-existed and interacted with each other. Is the situation worse now? The answer to this question might be yes. It is because of our immense population and our several activities that lead to flourishing of infections.

\section{Epidemiological and demographic transition}

Modern civilization dates back to approximately 10,000 BC. We are consuming earth's resources at a rate that is unsustainable. At around 8000 B.C., the global human population was 5 million. By 1 A.D., the population grew up to 200 million. It reached around 1 billion in the 1800 s during industrial revolution. The second billion was achieved in only 130 years (1930), the third billion in 30 years (1960), the fourth billion in 15 years (1974), and the fifth billion in only 13 years (1987). During the $20^{\text {th }}$ century alone, the population in the world has grown from 1.65 billion to 6 billion. It is projected to reach 14 to 18 billion by the end of the $21^{\text {st }}$ century. ${ }^{3}$ Today, SARS-CoV-2 has spread to 216 countries, areas and territories with nearly 6,310,635 infected cases and 3,74,723 deaths (as on 1 June, 2020), and has officially been termed as a "pandemic" by WHO on $11^{\text {th }}$ March 2020. Most of the COVID19-related deaths occurred in the USA, United Kingdom, Italy, France, Spain, Germany, Belgium, Brazil, Iran, Turkey, etc. ${ }^{4}$

In a global world with hyperconnected international travel and transport, points of entry (PoEs) - airports, ports, and ground crossings - could play a key role in the worldwide spread of diseases. The travel and transport sectors are indispensable part of public health emergency preparedness and response actions related to PoEs.

Most of the human respiratory infections caused by coronaviruses, including NL63, OC43, HKU1, and hCoV-229E, are mild in nature. However, in the past two decades, two beta-coronaviruses, Middle East respiratory syndrome coronavirus (MERS$\mathrm{CoV}$ ) and severe acute respiratory syndrome coronavirus (SARS$\mathrm{CoV}$ ), have severely infected more than 10,000 individuals, with mortality rates of $37 \%$ and $10 \%$, respectively. ${ }^{5}$ The fatality rate of COVID-19 is lower than both of these infections. ${ }^{6}$

Significance for public health

The pattern of infection of COVID-19 is unique and unpredictable. Previous studies have suggested that, similar to SARS-CoV, COVID-19 might interact with the angiotensin-converting enzyme 2 receptor in humans. Virus characteristics primarily define the risk of infection. These characteristics include the efficacy of the virus to spread, the severity of the disease after infection, and the efficacy and success rates of the available medical resources to contain the spread of the virus. With no vaccine or medication, community intervention becomes the most important response strategy against a pandemic. In a world of 7.8 billion people, countries have to be in a state of emergency preparedness to tackle emerging infectious diseases. 


\section{Unpredictable and dangerous virus}

The pattern of infection of COVID-19 is unique and unpredictable. Previous studies have suggested that, similar to SARSCoV, COVID-19 might interact with the angiotensin-converting enzyme 2 receptor in humans. The amino acid sequence of the nucleocapsid (N) protein of COVID-19 is $90 \%$ identical to that of SARS-CoV. COVID-19 might cross-react with the $\mathrm{N}$ protein antibodies of SARS-CoV; however, these antibodies might not provide cross-immunity. In addition, similar to SARS-CoV, SARS-CoV-2 might inhibit the RNA interference defense mechanism of the host organism. $^{7}$

Super-spreaders release significantly higher levels of viral particles compared to other infected individuals, which increases their chances of spreading the infection.

Coronaviruses are enveloped non-segmented positive-sense RNA viruses belonging to the family Coronaviridae and order Nidovirales. Coronaviruses target the upper respiratory tract, which leads to several ailments, including pneumonia. However, they might also affect the central nervous system that leads to longterm neurological damage. The RNA genome of coronavirus encodes for sixteen nonstructural proteins (nsp 1-16) with known or predicted RNA synthesis and modification functions, and they have also been suggested to trigger evolution of large genomes. ${ }^{8}$

Virus characteristics primarily define the risk of infection. These characteristics include the efficacy of the virus to spread, the severity of the disease after infection, and the efficacy and success rates of the available medical resources to contain the spread of the virus. With no vaccine or medication, community intervention becomes the most important response strategy against a pandemic. In a nutshell, COVID-19 risk can be defined as the risk of exposure versus risk of serious illness and death. The general risk factors of COVID-19 include advanced age and chronic medical conditions, such as diabetes, cardiac disorders, etc.

Reducing the number of emerging cases to a level where health-care facilities can adequately manage them is important to flatten the curve.

\section{Initial global response}

The world responded to COVID-19 pandemic with remarkable speed and resource mobilization. Within a matter of weeks, the causative agent for this pandemic was identified and sequenced by the Chinese scientists. Further genomic and clinical data were shared rapidly around the world. To date, various potential treatments have been proposed for this virus; however, none of the antiviral treatments have been approved. There is still no vaccine available for COVID-19. With ongoing research, it can be assumed that we would soon have a vaccine for SARS-CoV-2.

Social distancing is crucial in the regulation of the spread of the infection at community level. It plays an indirect role in prevention of the overwhelming of our health care systems by reducing the number of infection persons and the rate of infection. However, social distancing tests the degree of cooperation among humans. There is a correlation between perceived social connectedness and stress responses. More prominent effect could be observed on those who are already lonely or isolated. Better mental health is critical for better physical health. Prioritizing mental health is important during times of high stress. In the digital age and information superhighway, people are fortunate to live in an era where technology will allow us to see and hear our friends and family, even from a distance. Having said that, social distancing is not a permanent state, this is a moment in time. A multifarious approach is required, and involvement of community leaders is critical. Responsible electronic and print media also have an important role to play. Governments around the world are struggling with the question of how to reopen their economies while still containing the virus.

The COVID-19 could become endemic like HIV. It is here to stay, and it is impossible to predict that when this pandemic is supposed to control. This would take a massive effort even if a vaccine was found. It is still possible to control the virus, with effort. Strong and robust control of the virus is required in order to lower the assessment of risk. Everyone should contribute to stopping this pandemic.

Both life and livelihood are important. Deprivation is unacceptable. Shelter, food, healthcare and subsistence for weaker sections of population are the key challenges. Poor like migrant workers and casual labourers eat from garbage containers, which is a disgrace. Equally important is the care of internally displaced people and refugees. They need special attention.

Shaping the Future is critical. As schools remain closed during the pandemic, the education of many children for whom long distance learning is unavailable stands at great risk. Nations must do everything in their power to protect children from the dire consequences. Teens are feeling lonely and anxious in isolation. Poor children with limited access and illiterate/semi-literate parents are in serious trouble. They are losing out. This will impact them severely. As the pandemic pushes up levels of hunger among the global poor, governments must prevent devastating nutrition and health consequences for children missing out on school meals amid school closures.

The covid-19 pandemic could also lead to the spread of other preventable diseases. Economic recovery from COVID-19 will be much harder

\section{Indian scenario}

According to the Global Health Security Index 2019, India ranked $57^{\text {th }}$ among 100 countries on a scale to gauge preparedness for the outbreak of serious infectious diseases. Densely populated cities, low awareness, and unhygienic conditions can turn this outbreak into an epidemic in a matter of days. Shortage of health facilities, like hospital beds, rapid testing facilities, healthcare equipment, and quarantine facilities, would lay immense burden on an already over-stretched healthcare system. India with over 1.38 billion population must have robust and evidence-based health care management and action plans in place along with extraordinary monitoring, supervision, and continuous assessment to control this pandemic. While maintaining a zero-breach screening system must be a priority, it is equally important to ramp up the preparedness to meet any major outbreak if it occurs. India does not, at present, has the infrastructure and preparedness to face the challenges.

Community spread is already here. Containment facilities must be established right away, and human resource should be effectively trained in handling patients to minimize chances of virus spread. India has two major challenges, the size of population and limited resources. Rapid diagnosis is a very critical aspect in the collective response to COVID-19.

Some states in India are experimenting with containment zones. They could consider employing the Cluster Containment Strategy, which includes containment of the pandemic within a defined region with high rate of infection, and thus, preventing its spread to new areas. The Bhilwada model included a complete citywide curfew, an extensive follow-up and testing of its residents, and travel ban. This approach is considered to be more flex- 
ible and efficient. After implementation of this approach intensive risk communication campaign can be employed to encourage the citizens to stay in their homes for an initial period of 28 days, which can later be extended after subsequent risk assessment. The Government of India has decided to divide the entire country into three regions: red, orange, and green, based on the severity of the infection rates. However, the restrictions should be lifted in a staggered manner otherwise it can lead to a deadly resurgence.

India is far short of resources required to impede the pandemic. Nearly $41 \%$ of the population lack basic handwashing facility. India's health system cannot handle the upcoming turmoil, and the Government has done a great job containing the virus till now. This time should be used for consolidating patient-level data (clinical, labs, images, and outcomes) from the cases around the world (starting with India), and free access should be given to all the researchers. This data could have three potential applications: population-level predictions, patient-level predictions of outcomes, and quicker and more effective diagnosis.

Indian government ordered a nationwide sudden, severe and absolute lockdown on $24^{\text {th }}$ March, 2020, which is likely to end on 1 June 2020. However, owing the immediate imposition of the lockdown, proper planning could not be implemented. Migrant workers, daily wagers, manual laborers, and informal workers were left to fend for themselves. Hundreds of thousands had to walk over 400 kilometers to reach their homes in rural areas as they had no job or source of income in locked down metropolitan cities. Government should have arranged for shelter, food, and money for these poor people before ordering lockdown. Initially, India also lagged behind in testing. On an average 10,000 tests were conducted a day (now 15,000 per day), which was too small for a huge population of 1.38 billion. Contact tracing has also been weak. Health professionals do not have enough PPE. There is a shortage of ventilators and PPE all over the country. India's economy might be heading for its first full-year contraction in more than four decades. The lockdown would result in an $8 \%$ output loss and would also have indirect consequences, such as persistent fear community after the lockdown is over. It has been predicted that the gross domestic production would decrease by $0.4 \%$ and $0.1 \%$ till the end of this year and till March 2021, respectively. The lockdown will adversely affect the unorganized workforce as well as the banking and the corporate sectors. Overall, a precipitous recession is inevitable in India.

\section{What went wrong in the US?}

COVID-19 has been a disaster for the Americans. To date, the US has had the highest number of COVID-19-related cases and deaths than other countries. The American government's inaction has allowed the virus to spread inside its borders, and it has actively increased the risk to other countries. A faulty diagnostic kit rolled out by the American Centre for Disease Control and Prevention and slow approvals for other testing kits led to the disease spreading under the nose of the administration. The key problem with the kits was their negative control. CDC's test uses the polymerase chain reaction (PCR) assay to find tiny amounts of the SARS-CoV-2 genome in, say, a nose swab. To make sure a test is working properly, kits also include DNA unrelated to SARS-CoV2. The assay should not react to this negative control, but the CDC reagents did react with it at many, but not all, state labs. ${ }^{9}$

Because of slow initial response to the pandemic, tens of thousands more people might die. Millions more might lose their jobs, if the situation is not brought under control immediately. Social distancing and lockdown are inescapable necessities.

\section{Strengthening early diagnosis and effective mitigation}

The need of the day is to prioritize diagnostic tests and strengthen laboratory response network. Laboratories in all the major cities and government hospitals must be prepared to handle the diagnostic burden. Rapid diagnostic kits to identify the virus are currently being tested at different centers. Their testing, readiness, and approvals must be prioritized and sped up to ensure adequate diagnostic preparedness.

Effective communication is an indispensable tool. The Indian State of Kerala, which is the first state in India to encounter the disease, tackled the outbreak with a combination of swift measures, travel curbs, and effective communication. Timely dissemination of surveillance data, as well as effective communication with the healthcare system, allowed the state to contain the threat and prevent panic at the same time.

The governments must effectively communicate with all the stakeholders, including hospitals, health professionals, diagnostic facilities as well as the general public. A daily advisory, informing everyone about the situation, the need for precautions and travel restrictions will help keep a check on the disease at the community level. Professional management of health care delivery is an essential component of a successful program to contain this great challenge before us. Effective mitigation is an equally important component of the overall strategy.

\section{Limited options}

Clinical diagnosis is done on the basis of symptoms, chest imaging data, etc. However, the infection can be confirmed only by genetic testing of the samples obtained from the respiratory tract (such as throat swabs). Due to absence of an appropriate antiviral therapy, the patients are kept on supportive care. ${ }^{10}$ The suspected individuals must be isolated, until confirmation. If they are confirmed to be positive, then they must be quarantined. Furthermore, since asymptomatic transmission is also possible, it is necessary to test all the persons who have been in the areas with known transmission. In addition, the symptomatic mothers who are still breastfeeding must maintain proper hygiene before and after handling the baby.

It might be more efficient to obtain the samples from the suspected individuals, and then, send them home with appropriate instructions to maintain proper hygiene and to use respiratory masks until the test results come back. In case of shortage of hospital beds, the infected individuals who are not severely ill could be isolated and treated at their own homes. No hospitalization is needed unless the symptoms worsen. This strategy could be employed to save the hospital capacity for the needier people and prevent unintended infections to the otherwise healthy individuals while they await their test results at the hospital.

It is necessary to develop "point-of-care" diagnostic tests to rapidly test for 2019-nCoV infection in general population. Such tests could be widely used at checkpoints, in hospitals, and even in homes for suspicious persons. Moreover, they can also be used after every few weeks to check if any asymptomatic person is unintentionally spreading the virus. However, the individuals who are screened positive by these tests would still have to undergo laboratory tests for confirmation.

COVID-19 infection has led to the development of clusters of pneumonia with manifestations similar to those of SARS-CoV infection. COVID-19 infection might lead to the development of acute respiratory distress syndrome, admission to the ICU, and 
even death. The severity of the infection might also be associated with the development of cytokine storm syndrome.

The community-level of COVD-19 diagnosis is achievable via the aid of accurate and scalable point-of-care (POC) tests. ${ }^{11,12}$ Such POC tests could help in obtaining rapid diagnosis, which, in turn, could facilitate early detection of COVID-19 cases and adequate use of resources and implementation of preventive measures. Currently several such tests are in development phases. Different devices may be more suitable for diagnosing new cases on infection, while others, especially those that test for the presence of antibodies, are better suited to determining whether an individual has previously been infected. ${ }^{13}$

The rates of evolution and mutations in coronaviruses are very high, which makes it difficult to develop a vaccine against them. Even after quick diagnosis, decision of an adequate treatment is very challenging. In this regard, government support and funding play a crucial role because the processes like development of vaccine and antiviral agents require decades of research and assessment.

Another danger posed by this pandemic on people's lives arises from the disrupted supply of personal protective equipment (PPE) due to increase in demand, hoarding, misuse, panic buying, etc.

South Korea has shown an exemplary performance by significantly slowing the infection spread in the country with a population of 50 million without any lockdowns or other severe measures that were adopted by China. South Korea had a well-organized testing program that involved efficient isolation of the infected individuals as well as their contacts. In South Korea, more than 270,000 people have been tested, which indicates that more than 5200 tests per million individuals have been conducted there. In the US, only 74 tests per million individuals have been conducted. South Korea has demonstrated that diagnosis at large scale is key to epidemic control, along with case isolation and contact tracing. ${ }^{14}$ A speedy recovery would depend on most of the world getting the pandemic under control or achieving herd immunity.

\section{Economic impact}

Today we are confronted with an unprecedented crisis this world has never experienced before. The global economy has hit the worst recession due to the COVID-19 pandemic. The pandemic and society's responses to it are hitting the economies across the world simultaneously, suffering both a demand and a supply shock. The worst economic fallout since the Great Depression is anticipated. The International Monetary Fund (IMF), on $14^{\text {th }}$ April, 2020 , slashed growth forecast for the Indian economy, projecting a GDP growth of $1.9 \%$ in 2020 .

The COVID-19 has forced the general population around the world to rethink their daily lifestyle. In response to travel bans, school closures, and social distancing recommendations to limit the spread of the virus, many people have turned to digital tools to keep some semblance of normality. According to the International Labour Organization, the labor and economic crisis induced by the COVID-19 pandemic could lead to a rise in global employment from 5.3 million to 24.7 million. Overall, the global impact of this pandemic has been predicted to be around $0.1 \%$ to $0.4 \%$ of global GDP. ${ }^{15}$

The UN's Trade and Development Agency (UNCTAD) predicted that the economic triggered by COVID-19 might cost the global economy \$2 trillion in 2020. The most prominent adverse economic impact would be faced by the already heavily-indebted developing countries, particularly commodity exporters. ${ }^{16}$
In the long-term, the pandemic will reduce the productive capacity of the global economy. Greater moves toward selective self-sufficiency can be anticipated given supply the chain vulnerability.

COVID-19 pandemic might result in permanent shifts in political and economic powers. Many businesses that rely on human contact are already feeling an immediate impact, and we expect to see significant changes across all industries. Many countries will have difficulty in recovering from the present scenario. In the aftermath of pandemic, people will rush to regain their sense of equilibrium and normalcy. The nations that responded early to the pandemic, such as Korea, Taiwan, Singapore, Hong Kong, and China, have emerged as victors, and will write the history.

\section{State of children}

According to UN report regarding the impact of COVID-19 on children, COVID-19 can cause deaths of hundreds of thousands of children. Furthermore, in the aftermath of this crisis, 42-66 million more children could fall into poverty. The impact of this pandemic could essentially reverse the recent improvements in infant mortality. The children living in slums, refugee camps, and regions of active conflicts would suffer the most from this crisis. Furthermore, UNICEF has reported that practically all the schools have been closed globally. This has directly affected the education and indirectly affected the nutrition of the children. With schools' shutdown, around 310 million children who are dependent on the school meals now miss a vital part of their nutrition. ${ }^{17}$

Another concern is delay in routine immunization of children that makes them vulnerable to disease. Once the pandemic is over, every child in need must be vaccinated. Unprecedented international solidarity is crucial for children and humanity.

\section{Emerging perspectives}

As scientists strive to understand the respiratory COVID-19 disease and its causal agent, the need for aggressive and fruitful research on the subject is the need of the hour. During SARS outbreak, South Korea had learned the importance of being prepared. With emergence of novel coronavirus, Korea Centers for Disease Control and Prevention (KCDC) raced to develop commercial test kits in cooperation with industrial manufacturers. They also designed the following protocol.

Severely ill patients are first hospitalized. The individuals with moderate symptoms are sent to repurposed facilities for basic medical support. If they are tested negative twice, then they are released. The individuals with minimal symptoms are advised to quarantine themselves for two weeks.

The COVID-19 pandemic is tackled by various strategies. Some of these strategies include minimizing human-to-human transmissions to reduce secondary transmissions, identifying and isolating infected individuals at early stages, minimizing transmission via animals, elucidating the factors that affect clinical severity, accelerating the development of diagnostic kits and vaccines, communicating the risk factors to arise awareness among the communities, minimizing the spread of misinformation, and minimizing the economic and social impacts.

The four key stages of any pandemic include investigation, recognition, initiation, and acceleration. Maximum number of infected individuals is found at the end of the acceleration phase. This stage is finally followed by a deceleration stage, in which the 
number of infected individuals decreases.

To reduce community spreads, mass gatherings must be discouraged. Increased incidences of community spread would lead to increase in the number of individuals who would need medical care. This would surely lead to overloading of healthcare systems due to increased hospitalizations and deaths.

Currently, the primary global focus is on minimizing the spread of the virus. Everyone can do their part to help us respond to this emerging public health threat.

The early studies on COVID-19 from Hubei province in China have reported case series of hospitalized COVID-19 patients and could provide information regarding the severe end of the disease spectrum. Based on these reports, about $81 \%$ of COVID-19 patients suffer from only mild fever and do not require hospitalization. There have note bee extensive reports on such patients yet. ${ }^{18}$

The absence of adequate PPE leads to a much higher risk of exposure to healthy individuals who are either close to infected persons or are working in healthcare systems. Putting high-risk persons under quarantine with minimal public interactions could play a key role in the spread of SARS-CoV-2. Clinicians should work in association with public health departments on a case-bycase basis.

Vaccine development is crucial. A faster way has to be developed to identify antibodies that neutralize the virus. Researchers have to generate lung tissue models called organoids to test candidate drugs for COVID-19 and harness the power of individual genomes to identify variants that raise or lower people's risk of serious illness when infected by SARS-CoV-2. More than 90 vaccines for the SARS-CoV-2 are at various stages of development. Some six groups have already begun injecting formulations into volunteers in safety trials; others have started testing in animals.

We need a drug or combination of drugs that work. Remdesivir has generated hope. It is an antiviral and works by attacking an enzyme that a virus needs in order to replicate inside our cells. Experts believe that Remdesivir has a clear-cut, significant, positive effect in diminishing the time to recovery thus can block this virus. It may prove to be a magic bullet.

\section{Preparedness}

COVID-19 pandemic management requires a planned proactive strategy for efficient prevention and control of the virus. Life threatening situations demand extraordinary response and more stringent measures to contain the pandemic. Extreme social distancing and avoiding public places, like theatres, clubs, and pubs, can flatten the pandemic curve and peak. It may place huge pressure on the society. We are living through an unprecedented biopsychosocial crisis. We find ourselves in a time of great economic, social, and medical uncertainty. The human cost of COVID pandemic could be extraordinary. It endangers thousands of lives. The pandemic has catalysed the development of novel coronavirus vaccines across the biotech industry. Future pandemics are likely to happen more frequently, spread more rapidly, have greater economic impact and kill more people if we are not extremely careful about the possible impacts of the choices we make today. Nations have to build a healthier world by focusing on the shared good health of human and nature.

COVID pandemic has exposed the Health Systems weaknesses and deficiencies of most if not all countries. Science is failing all over. Health care workers around the world have shown unimaginable bravery, dedication and commitment to helping others during the pandemic, often at great risk to themselves.

Countries like New Zealand, South Korea, Taiwan, Singapore, Hong Kong, Vietnam. have done exceptionally well to contain the spread of COVID-19. New Zealand has shown the way. Decisive action, going hard and going early, helped to stamp out the worst of virus. Testing was done widely.

The COVID-19 pandemic highlights the need to protect people from health emergencies, as well as to promote universal health coverage and healthier populations to keep people from needing health services through multisectoral interventions like improving basic hygiene and sanitation. The COVID-19 pandemic demands action on many fronts, from prevention to testing to treatment. We need to create simple, cheap, more accessible testing for SARSCoV-2, the virus that causes COVID-19, both in humans and on various surfaces. Scientists have to develop a faster way to identify antibodies that neutralize the virus. It is crucial to identify any antibodies that inadvertently help the virus enter cells so researchers who are developing vaccines can avoid including those antibodies. There are some opportunities to pursue other interventions in the short- and long-term that could save lives. Till proper treatment or vaccine is made available, people have to rely on social distancing, contact tracing, self-isolation, and other measures.

To end this pandemic, we must act in solidarity. Nations will have endemic SARS-CoV-2 infection for the foreseeable future. A structured and well-coordinated approach is critical for success. Global crisis needs global action. Collaboration among the researchers, clinicians, industry, and government is critical for efficient disease diagnosis, improved basic and clinical research, devising of appropriate countermeasures, timely manufacture of relevant drugs and vaccines, and adequate therapeutic management of infected individuals.

Pandemic will disproportionally harm the poor globally. This is because they lack access to health care and have lower immunity. Nation's responses to existing inequalities is critical. Moreover, safety of health workers must be strengthened.

World is facing such an unprecedented global crisis. Every day, refugee and migrant children, and those affected by conflict face major threats to their safety and well-being. The pandemic is deepening inequalities, and millions more women and girls now risk losing the ability to plan their families and protect their bodies and their health.

Group upon group of terrified, starving, exhausted people leave the cities they built, carrying with them precious items packed into sacks. Some clothes, biscuit packets, bread. They flee on foot, bicycles, hitch a ride on goods-laden trucks, hungry, thirsty and tired, to rest under the shade of a tree or a school converted into a shelter for migrants.

Countries must address the fragilities, inequalities and gaps in social protection that have been so painfully exposed, and place women and gender equality front and centre. Nations must keep food supply chains alive and protect the most vulnerable. What the world needs right now is solidarity along with bold and courageous leadership guided by science, data, ethics and empathy. Recovery from COVID can steer the world toward a safer, healthier, more sustainable and inclusive path.

The pandemics lead to communities or individuals being stigmatized. It is not only irresponsible but unethical to associate a virus and the disease it causes with a specific place or members of a particular race or community. It is tragic and unacceptable. The stigma must stop now. Every nation and every individual, is experiencing the societal strain of this disease in new and powerful ways. 


\section{Through the storm}

Today, to efficiently tackle the global crisis of COVID-19 pandemic, several countries have employed a combination of containment and mitigation strategies for proper treatment of the infected and to prevent the spread of the infection. These strategies include self-isolation, quarantine, contact tracing, public health management, improving health facilities, etc. ${ }^{19}$

In China, where severely aggressive strategies were employed, minimal level of nosocomial transmission was observed. China's epidemiological records showed that most of the viral transmissions occurred within the family clusters. ${ }^{20}$ This data indicated that the viral transmission mainly occurred via direct contact or contact with fomites in close proximity to the infected individuals.

Nations need to rapidly and robustly increase their preparedness, readiness, and response actions. Intensified case finding and contact tracing are crucial. Other than case and contact finding, containment or other measures that aim to delay the onset of patient surges where feasible; and measures such as public awareness, promotion of personal protective hygiene, preparation of health systems for a surge of severely ill patients, stronger infection prevention and control in health facilities, are of paramount importance.

Surveillance and rapid testing to identify both asymptomatic and symptomatic cases (contact tracing) is required, followed by quarantine and appropriate treatment. Contract tracing can play a key role in interrupting the pathways of future transmissions. Moreover, pool testing in non-hotspot areas can be beneficial.

Moreover, the large numbers of infections reported among health workers need urgent attention. Risk of transmission must be substantially minimised if not prevented. Their safety is absolutely essential. Solidarity at national and global levels is need of the hour. Nations have a responsibility to look after weaker sections of society. Supply chain must be maintained (PPE, ventilators, oxygen, kits, etc.) equitably at global and national levels. No failures in this regard are acceptable. Furthermore, over 1.6 billion children are out of school. Novel methods have to be developed to ensure that education does not suffer.

\section{Tackling chronic disease burden}

It is widely believed that during the COVID pandemic treatment suffers. Cancer patients get either neglected or do not have access to quality healthcare and medicines. Similarly, patients with Diabetes have difficulties in managing the disease at an appropriate health facility. Patients who need renal dialysis suffer because of non-availability of medical care or transportation facility. Pregnant ladies from rural areas or even from towns face immense problems so far conducting delivery (normal or Caesarean Section) is concerned. Those who have cardiac disease are unable to reach hospitals for any prompt medical attention.

\section{Ethical issues}

COVID pandemic is turning us into a cruder society. Fear of virus, else seeing the death coming so near, is forcing us to think of individual safety rather than collective security and dignity. Stigma is a curse and needs to be discouraged with full might. What is required is burying dead with dignity, giving human face to lockdown, citizen-state engagement, creating a social-security net for poor. Moreover, this is the time of reason and science not rhetoric. The number of women unable to access family planning, facing unintended pregnancies, gender-based violence and other harmful practices could skyrocket by millions of cases in the months ahead.

It is widely believed that during the pandemic treatment suffers. Patients with diseases like cancer, diabetes, renal failure, $\mathrm{CAD}$ and pregnant women need special attention. As the pandemic pushes up levels of hunger among the global poor, governments must prevent devastating nutrition and health consequences for children missing out on school meals amid school closures. Initial response to this global crisis by some countries has been so feeble and spotty. The response serves as a critical reminder for why strong government leadership is needed during a global crisis.

Many basic facts about the virus remain unknown. Viruses have no nationality so there is no place for discrimination on the basis of race, religion, caste, etc. We have to work all together to defeat the virus. Empathy and simple kindness are essential powerful tools of national recovery.

\section{Emerging trends}

To date, no specific therapy has been scientifically proven to reduce mortality. tackling this global crisis. Scientists are working hard to develop vaccines and treatments to slow the pandemic, and reduce morbidity and mortality. Drugs are still being tested in clinical trials to see if they are effective against COVID-19. The medications have to be safe for this particular use. The coronavirus vaccines in various stages of development, across the world include: Fusogenix DNA vaccine by Entos Pharmaceuticals, ChAdOx1 nCoV-19 by University of Oxford, Gimsilumab by Roivant Sciences, AdCOVID by Altimmune, TJM2 by I-Mab Biopharma, Coronavirus vaccine by Medicago, AT-100 by Airway Therapeutics, TZLS-501 by Tiziana Life Sciences, OYA1 by OyaGen, BPI-002 by BeyondSpring, Altimmune's intranasal coronavirus vaccine, INO-4800 by Inovio Pharmaceuticals and Beijing Advaccine Biotechnology, NP-120 (Ifenprodil) by Algernon Pharmaceuticals, APN01 by University of British Columbia and APEIRON Biologics, mRNA-1273 vaccine by Moderna and Vaccine Research Center, Avian Coronavirus Infectious Bronchitis Virus (IBV) vaccine by MIGAL Research Institute, TNX-1800 by Tonix Pharmaceuticals, Brilacidin by Innovation Pharmaceuticals, Recombinant subunit vaccine by Clover Biopharmaceuticals, Vaxart's coronavirus vaccine, CytoDyn-leronlimab, Linear DNA Vaccine by Applied DNA Sciences and Takis Biotech, BXT-25 by BIOXYTRAN to treat late-stage acute respiratory distress syndrome (ARDS), MERS CoV vaccines for coronavirus, Novavax's MERS coronavirus vaccine candidate, Inovio Pharma's INO-4700, etc.

The novel coronavirus drugs in various stages of development globally include: Remdesivir (GS-5734) by Gilead Sciences, Actemra by Roche to treat coronavirus-related complications, Biocryst Pharma's Galidesivir, a potential antiviral for coronavirus treatment, Regeneron's REGN3048-3051 and Kevzara, SNG001 by Synairgen Research, AmnioBoost by Lattice Biologics, etc. Remdesivir has generated some hope, a new antiviral, as well as a drug that inhibits the action of interleukin-6. Remdesivir works by attacking an enzyme that a virus needs in order to replicate inside our cells. Experts believe that remdesivir has a clear-cut, significant, positive effect in diminishing the time to recovery thus can block this virus. It may prove to be a magic bullet. China has approved the use of Favilavir, an anti-viral drug, as a treatment for coronavirus. The drug has reportedly shown efficacy in treating the disease with minimal side effects. However, the sample size of the 
trial has been small. It can take a decade for a new compound to go from initial discovery to the marketplace. Many compounds never even make it that far.

It is crucial to identify any antibodies that inadvertently help the virus enter cells so researchers who are developing vaccines can avoid including those antibodies. There are some opportunities to pursue other interventions in the short- and long-term that could save lives.

The pandemic demands action on many fronts, from prevention to testing to treatment. We need to create simple, cheap, more accessible testing for SARS-CoV-2. A faster way has to be developed to identify antibodies that neutralize the virus. Over 100 vaccines for the SARS-CoV-2 are at various stages of development, and at least six are being tested for safety in people. The biggest challenge is to determine which vaccine is effective if not ideal. Reason and science have to guide us.

There is urgent need to critically appraise evidence in deciding how to treat patients. Remdesivir has generated hope. It is an antiviral and works by attacking an enzyme that a virus needs in order to replicate inside our cells. Experts believe that Remdesivir has a clear-cut, significant, positive effect in diminishing the time to recovery thus can block this virus. It may prove to be a magic bullet.

Scientists and researchers across the globe are racing against the time to develop a vaccine for the novel COVID-19. More than 100 potential vaccines are being developed, including several in clinical trials, but experts have underscored the difficulties of finding vaccines that are effective against coronaviruses. Collaborating across the healthcare innovation ecosystem to making the impossible possible, is need of the hour. Researchers and scientists have been relentlessly working to develop an investigational antiviral compound, a vaccine to prevent infection as well as evaluating other therapies that have scientific potential to help infected patients fight the virus. Both antivirals and vaccines will be valuable tools in combating COVID-19.

\section{Lessons learnt}

The countries that effectively managed COVID-19 disease by implementing strong measures in place included were Taiwan, Vietnam, New Zealand, South Korea, and Singapore. Each of these countries imposed travel restrictions, quarantines, social distancing, and heightened hygiene. In Singapore, three university hostels were converted into quarantine facilities and people were compensated for any workdays lost. Officials then began aggressively tracing all contacts of known infected people, using data from transport companies, hotels, and CCTV footage. Though large gatherings have been suspended, schools and offices have remained open to limit the outbreak's social and economic costs. However, all students and employees undergo daily health checks and temperature screenings.

Taiwan screened the passengers on planes after landing. It later halted the flights incoming from Wuhan and other parts of China after its first imported case. It used home quarantines more extensively than others despite the availability of state facilities. Disobeying quarantine orders also drew a penalty of up to INR 2,500,000. Mass gatherings and religious services were banned.

Hong Kong's strategy focused on limiting the local transmission rather than preventing infected people from entering the city boundaries. It expanded its existing temperature screening facilities at entry points and asked local clinics to report any patient showing symptoms and with a travel history to Wuhan. All the people crossing the border from China were placed under manda- tory 14-day quarantine. Vacant, newly constructed public housing buildings were converted into quarantine facilities. Social distancing was extensively implemented - large-scale events were canceled, schools were closed until April, and civil servants were asked to work from home for a month. In South Korea everyone was tested; however, in Italy sick people were tested. Number of COVID-19 cases has dropped in South Korea. It necessitates the need for increase in number of tests. To minimize the impact of future epidemics, laboratories and epidemiological capacities must be established at regional and national levels. Improved surveillance could facilitate higher degree of prevention and treatment of the disease. New Zealand has shown the way. There is no widespread, undetected community transmission. Decisive action, going hard and going early, helped to stamp out the worst of virus. Testing was done widely. An effective or ideal vaccine may take more than a year to develop. Even after the vaccine has been developed, its effectiveness, side effects, availability, and accessibility will be a larger issue. The world community will see pockets of resurgence of disease. Moreover, antibody testing can be used for public testing to determine who is immunized and free to work and intermingle. Older people will have to be protected during this exercise by lockdown and social distancing.

The COVID-19 could become endemic like HIV. It is here to stay, and it is impossible to predict that when this pandemic is supposed to control. This would take a massive effort even if a vaccine was found. It is still possible to control the virus, with effort. Strong and robust control of the virus is required in order to lower the assessment of risk. Everyone should contribute to stopping this pandemic. Both life and livelihood are important. Deprivation is unacceptable. Shelter, food, healthcare and subsistence for weaker sections of population are the key challenges. Poor like migrant workers and casual labourers eat from garbage containers, which is a disgrace. Equally important is the care of internally displaced people and refugees. They need special attention.

Shaping the Future is critical. As schools remain closed during the pandemic, the education of many children for whom long distance learning is unavailable stands at great risk. Nations must do everything in their power to protect children from the dire consequences. Teens are feeling lonely and anxious in isolation. Poor children with limited access and illiterate/semi-literate parents are in serious trouble. They are losing out. This will impact them severely. As the pandemic pushes up levels of hunger among the global poor, governments must prevent devastating nutrition and health consequences for children missing out on school meals amid school closures.

\section{Conclusions}

In a world of 7.8 billion people, countries have to be in a state of emergency preparedness to tackle emerging infectious diseases. It needs an aggressive and focused action, with coordination among all departments of the government - health, security, finance, commerce, transport, trade, information, diplomacy, etc. Global EID threats require an organized global response including adequate communication, epidemiology, behavioral science, laboratory science, adequate public health infrastructure, increased surveillance, and primary prevention. Efficient prevention and regulation of problems would require elucidation of causal factors of disease, disability, and premature death. Under such evolutionary changes, the future trends cannot be predicted by the extrapolation of current scenarios. Instead accurate prediction can be made by scientists and professionals by applying the current data to various possible scenarios. 
An optimum harmony can be maintained between humans and environment by improving human resistance, environmental safety and adequate healthcare systems. One might consider infectious diseases as an imbalance between humans and environment, caused by the dominance of microbes. The economic, social, administrative, and legal forces must work together towards better human health.

The approach to tackle this pandemic has to be solidly evidence-informed. Balancing act between public health crisis and complex societal implications is an inescapable necessity. In these unprecedented and critical times, the global community has a moral responsibility to jointly fight this dreaded virus that can otherwise lead to millions of avoidable deaths. The private sector also has an important role to play in efforts to fight this pandemic. During these most difficult times in human history, nations need to ensure that the needs of the general public are met and the businesses can resume normal operations.

After the pandemic is over, structure of the society will change. New geopolitical and socioeconomic dynamics will emerge, values will change, and our lives and habits will also change. Nations will have to prioritise their expenditure. Weaker sections of society will need all the possible help. However, regardless of the devastation caused by the crisis, human population will prevail. Humanity will still find the strength to recover. Investing in public health, preparedness, and relying on science will bring a better future.

Correspondence: Prof. S. A. Tabish, Sher-i-Kashmir Institute of Medical Sciences, Srinagar, India. E-mail: amintabish@gmail.com

Key words: COVID19; pandemic; emerging infectious diseases; social distancing; socioeconomic impact.

Conflict of interest: The author declares no potential conflict of interest.

Funding: None.

Received for publication: 27 April 2020.

Accepted for publication: 26 May 2020.

(Copyright: the Author(s), 2020

Licensee PAGEPress, Italy

Journal of Public Health Research 2020:9:1786

doi:10.4081/jphr.2020.1786

This work is licensed under a Creative Commons Attribution

NonCommercial 4.0 License (CC BY-NC 4.0).

\section{References}

1. Tabish SA. Recent trends in emerging infectious diseases. Int J Health Sci (Qassim) 2009;3:5-8.

2. Tabish SA, Nabil S. The future of humanity and microbes: Impact of emerging infectious diseases on global health economies. IJSR 2015;4:2427-42.

3. Worldometers [Internet]. Available from: https:/www.worldometers.info/world-population/
4. WHO. Coronavirus disease 2019 (COVID-19) Situation Report. https:/www.who.int/emergencies/diseases/novelcoronavirus-2019

5. Cascella M, Rajnik M, Cuomo A, et al. Features, evaluation and treatment coronavirus (COVID-19). StatPearls [Internet].

6. Fauci AS, Lane HC, Redfield RR. Covid-19-navigating the uncharted. N Engl J Med 2020. doi: 10.1056/NEJMe2002387

7. Kannan S, Shaik Syed Ali P, Sheeza A, Hemalatha K. COVID19 (Novel Coronavirus 2019) - recent trends. Eur Rev Med Pharmacol Sci 2020;24:2006-11.

8. Denison MR, Graham RL, Donaldson EF, et al. An RNA proofreading machine regulates replication fidelity and diversity. RNA Biol 2011;8:270-9.

9. Cohen J. The United States badly bungled coronavirus testing - but things may soon improve. Sciencemag.org. [Internet]. Published Feb 28, 2020

10. Wu Z, McGoogan JM. Characteristics of and important lessons from the coronavirus disease 2019 (COVID-19) outbreak in China: summary of a report of 72314 cases from the Chinese Center for Disease Control and Prevention. JAMA 2020. doi: 10.1001/jama.2020.2648

11. Wang C, Horby PW, Hayden FG, Gao GF. A novel coronavirus outbreak of global health concern. Lancet 2020;395:470-3.

12. Nguyen T, Bang DD, Wolff A. 2019 Novel coronavirus disease (COVID-19): Paving the road for rapid detection and point-ofcare diagnostics. Micromachines 2020;11:306.

13. Green K, Graziadio S, Turner P, et al. Molecular and antibody point-of-care tests to support the screening, diagnosis and monitoring of COVID-19. cebm.net. [Interenet]. Available from: https://www.cebm.net/covid-19/molecular-and-antibody-point-of-care-tests-to-support-the-screening-diagnosisand-monitoring-of-covid-19/

14. Normile D. Coronavirus cases have dropped sharply in South Korea. What's the secret to its success? Sciencemag.org [Internet]. Available from: https://www.sciencemag.org/ news/2020/03/we-are-social-species-how-will-social-distancing-affect-us

15. Abiad A, Arao RM, Dagli S, et al. The economic impact of the COVID-19 outbreak on developing Asia. Asian Development Bank [Internet]. Available from: https://www.adb.org/publications/economic-impact-covid19-developing-asia

16. World Economic Forum and United Nations. This is how much the coronavirus will cost the world's economy, according to the UN. [Internet]. Available from: https://www.weforum. org/agenda/2020/03/coronavirus-covid-19-cost-economy2020-un-trade-economics-pandemic/

17. UN Policy Brief. The impact of COVID 19 on children. United Nations [Internet]. Available from: https://www.un.org/ sites/un2.un.org/files/policy_brief_on_covid_impact_on_children_16_april_2020.pdf

18. Zhou F, Yu T, Du R, et al. Clinical course and risk factors for mortality of adult inpatients with COVID-19 in Wuhan, China: a retrospective cohort study. Lancet 2020;395:1054-62 .

19. Bedford J, Enria D, Giesecke J, et al. COVID-19: towards controlling of the pandemic. Lancet 2020;395:1015-8.

20. WHO. Report of the WHO-China Joint Mission on Coronavirus Disease 2019 (COVID-19). Available from: https:/www.who.int/docs/default-source/coronaviruse/whochina-joint-mission-on-covid-19-final-report.pdf 